\title{
Arborescences
}

Revue d'études françaises

\section{La norme orale en français laurentien Introduction}

\section{Marie-Hélène Côté et Anne-José Villeneuve}

Numéro 7, décembre 2017

La norme orale en français laurentien

URI : https://id.erudit.org/iderudit/1050965ar

DOI : https://doi.org/10.7202/1050965ar

Aller au sommaire du numéro

Éditeur(s)

Département d'études françaises, Université de Toronto

ISSN

1925-5357 (numérique)

Découvrir la revue

Citer ce document

Côté, M.-H. \& Villeneuve, A.-J. (2017). La norme orale en français laurentien :

introduction. Arborescences, (7), 1-16. https://doi.org/10.7202/1050965ar

Tous droits réservés @ Département d'études françaises, Université de Toronto, Ce document est protégé par la loi sur le droit d’auteur. L’utilisation des d'utilisation que vous pouvez consulter en ligne.

https://apropos.erudit.org/fr/usagers/politique-dutilisation/ 


\section{SOMMAIRE}

1 Marie-Hélène Côté, Université de Lausanne

Anne-José Villeneuve, Université de l'Alberta Introduction

17 Davy Bigot, Université Concordia

Regard rétrospectif sur la norme du français québécois oral

33 Marie-Hélène Côté, Université de Lausanne

Wim Remysen, Université de Sherbrooke

Le "bon usage du français au Québec» selon le Multidictionnaire de la langue française: le cas de la prononciation

49 Anne-José Villeneuve, Université de l'Alberta

Normes objectives et variation socio-stylistique: le français québécois parlé en contexte d'entrevues télévisées

67 Monelle Guertin, Université du Québec à Montréal

Variation sociophonétique dialectale et stylistique: quelle est la langue cible en français langue seconde à Montréal?

90 Suzie Beaulieu, Université Laval

Leif French, Sam Houston State University

Samuel Gagnon, Université Laval

I'veulent-tu parler comme nous-autres? Opinions d'apprenants sur la forme interrogative totale en français laurentien 


\title{
La norme orale en français laurentien
}

\author{
Marie-Hélène Côté, Université de Lausanne \\ Anne-José Villeneuve, Université de l'Alberta
}

\section{Introduction}

Un norme linguistique est porteuse de divers pouvoirs symboliques et sociaux. Dans le marché linguistique de la francophonie, les variétés laurentiennes dominent les discours et l'imaginaire collectif. À l'échelle mondiale, la province de Québec est l'un des membres canadiens siégeant à l'Organisation Internationale de la Francophonie et ce, depuis au moins 40 ans ${ }^{1}$. Au Canada et aux États-Unis, la plupart des îlots ${ }^{2}$ francophones nord-américains, mises à part les zones de peuplement acadiens, partagent avec le Québec des racines canadiennes-françaises, une origine qui demeure évidente dans les traits linguistiques communs (affrication des occlusives dentales, marqueur de discours là, pronoms pluriels composés en autres, etc.).

Face à ce statut relativement privilégié au plan sociolinguistique, on peut s'étonner que le débat sur la «qualité de la langue» et «la» norme continue de faire couler autant d'encre au Québec et dans le domaine laurentien ${ }^{3}$, chez les spécialistes et dans le grand public (Bédard et Maurais 1983, CajoletLaganière et Martel 1995, Martel et Cajolet-Laganière 1996, Bouchard 1998, entre autres). Au sein de ce débat, certains s'interrogent particulièrement sur la variété orale à privilégier dans l'enseignement et dans les médias. Comment peut-on envisager à l'oral le "bon» usage dans le domaine laurentien? Comment peut-on concilier, d'une part, le pouvoir apparent (angl. "overt prestige») des groupes sociaux privilégiés et de la langue écrite, d'autre part, les pouvoirs latents (angl. "covert prestige») des groupes de locuteurs ordinaires?

Dans ce numéro d'Arborescences: revue d'études françaises, nous nous intéressons à la norme orale en français laurentien, en mettant l'accent sur la prononciation et la morphosyntaxe (la "grammaire») du français parlé au Québec. Nous examinons dans ce texte d'ouverture chacune des trois composantes de la thématique: norme(s), oralité et domaine laurentien. La section 1 définit la notion de norme, en tentant de décrire les liens qu'elle entretient avec les divers usages, et notamment avec «le bon usage». Dans la section 2, nous mettons en lumière quelques aspects permettant de distinguer norme orale et norme écrite, en démontrant qu'une norme orale basée principalement sur l'écrit sera nécessairement lacunaire. Enfin, dans la section 3, nous nous focalisons sur la variation diatopique (ou dialectale) en mettant l'accent sur la norme laurentienne. Nous concluons par une présentation des contributions

1. Y siègent un représentant national et deux provinces - le Québec et le Nouveau-Brunswick - depuis les années 1970 , de même que l'Ontario, qui a obtenu le statut d'observateur en 2016.

2. L'usage universitaire n'étant toujours pas fixé quant à la mise en application des rectifications orthographiques proposées en 1990, le présent texte emploie l'orthographe traditionnelle. Pour une présentation de l'orthographe rectifiée, voir la Banque de dépannage linguistique de l'Office québécois de la langue française: http://bdl.oqlf.gouv.qc.ca/bdl/ gabarit_bdl.asp?id=3195.

3. Nous évitons l'expression «français canadien» (Rochet 1994) dans le but de reconnaître l'existence non pas d'une seule mais bien de deux grandes variétés de français traditionnellement parlées au Canada: l'acadien, parlé dans les provinces maritimes, et le laurentien, regroupant les français du Québec (à l'exception des zones de peuplement acadien à l'est de la province), de l'Ontario, de l'Ouest canadien et des diasporas aux États-Unis. 
scientifiques regroupées dans ce numéro et par une remise en question de la légitimité d'une norme laurentienne unique.

\subsection{Une norme ou des normes? Nuancer la terminologie autour de la notion de norme}

Avant d'aborder la question de la norme linguistique à l'oral, il faut d'abord définir ce qu'est une norme. On se trouve alors devant un premier constat: l'hésitation constante entre le normatif, qui relève de l'évaluation, et le normal, qui relève des usages les plus fréquents au plan statistique. Cette vacillation est évidente dans le Petit Robert, tant pour la définition générale que pour celle associée au champ de la linguistique:

1. LITTÉR. Type concret ou formule abstraite de ce qui doit être.

2. État habituel, conforme à la majorité des cas [cf. la moyenne, la normale].

[...]

4. LING. Ce qui, dans la parole, le discours, correspond à l'usage général [...]. - Usage d'une langue valorisée comme «bon usage» et rejetant les autres, jugés incorrects.

L'ambiguïté entourant la notion de norme se dégage également des deux définitions offertes par le Grand dictionnaire de linguistique et des sciences du langage:

1. On appelle norme un système d'instructions définissant ce qui doit être choisi parmi les usages d'une langue donnée si l'on veut se conformer à une certain idéal esthétique ou socioculturel. La norme, qui implique l'existence d'usages prohibés, fournit son objet à la grammaire normative ou grammaire au sens courant du terme.

2. On appelle aussi norme tout ce qui est d'usage commun et courant dans une communauté linguistique [...]. (Dubois, et al. 2007: 330)

On y voit ainsi l'incertitude entre une norme au singulier (le normatif), et des normes au pluriel (le normal). Alors que la première émane d'une approche prescriptive, subjective de la langue, hiérarchisant certains usages vis-à-vis des autres, les secondes envisagent plutôt la langue selon une approche objective, voire statistique, où les usages les plus répandus se constituent en autant de normes plurielles. Suivant la typologie proposée par Moreau (1997), on opposera alors normes prescriptives, objectives, fantasmées, etc.

Aborder la norme orale, dès lors que l'on choisit de parler de norme au singulier, implique la sélection d'usages émanant d'un groupe de locuteurs perçu comme porteur d'un certain prestige. Toutefois, cette évaluation n'implique pas nécessairement un processus de standardisation, comme le fait remarquer Gadet (1995: 19), à la suite de Bloomfield (1927: 439) :

Il n’y a pas de société qui n'introduise de la différenciation entre productions linguistiques, en une évaluation qui prend la forme de jugements de valeurs assortis de hiérarchisations. Et ce phénomène est partiellement indépendant du processus de standardisation ou de normalisation, puisque [...] on le constate aussi dans des sociétés sans norme institutionnalisée.

Pour les besoins du présent numéro, nous adoptons une vision du concept de norme où les usages (aspect objectif) et l'évaluation que les locuteurs font de ces usages (aspect subjectif) sont considérés 
conjointement. Nous portons donc notre attention principalement sur les usages qui font l'objet d'évaluations positives au sein de la communauté linguistique (normes objectives valorisées, dominantes ${ }^{4}$ ).

Nous tentons d'adopter une approche ascendante («bottom-up») partant des usages réels pour aller vers le modèle plutôt que d'imaginer a priori un modèle descendant («top-down») auquel sont supposés se conformer les locuteurs. Cette décision reconnaît donc d'emblée le lien étroit qu'entretient la norme (désormais au singulier) à la variation diastratique (ou sociale) et diaphasique (ou stylistique) ${ }^{5}$. Comme Labov (1972) définit une communauté linguistique non pas à partir de comportements linguistiques identiques, mais plutôt en fonction d'une participation à des normes linguistiques partagées, une norme est un point de convergence ou de consensus au sein d'une communauté linguistique. Ainsi, devant un trait linguistique évalué comme un marqueur familier (Figure 1), la stratification sociale et le continuum stylistique montrent des comportements qui convergent vers un point défini, vers une norme que visent les membres des diverses couches sociales quand, selon l'approche de Labov (1972), le niveau d'attention qu'ils portent à leur parler augmente.

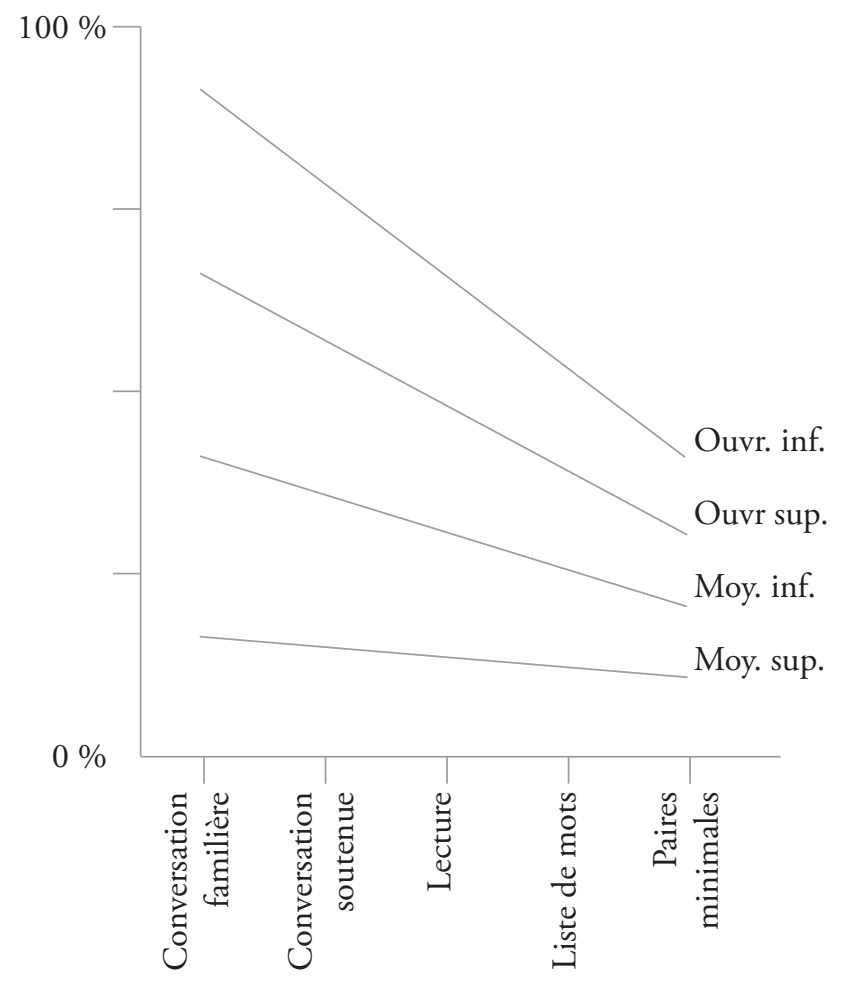

Figure 1: Lien entre variation diaphasique et stratification sociale (adapté de Bell 1984: 153)

4. Rappelons ici qu'on oppose à ces normes dominantes des normes dominées (angl. 'covert norms'), qui font l'objet d'évaluations visant avant tout la solidarité plutôt que le prestige social. Par exemple, une Québécoise pourrait opter pour le marqueur de conséquence (ça) fait que [fak, fєk] dans un échange avec un francophone du nord de l'Ontario afin de marquer leur appartenance partagée à la francophonie canadienne.

5. Nous évitons ici de parler de registre de langue, employé communément dans le grand public, en raison de la consécration du terme en sociolinguistique. Un registre fait habituellement référence à une performance linguistique régie par des conventions souvent d'ordre professionnel : langue employée par les annonceurs de sport (Ferguson 1983) ou avec les bambins (Cooper et Aslin 1990), par exemple. 
Pour le français en usage au Québec, les critères de sélection de la variété ciblée semblent plutôt clairs dans l'esprit collectif, comme en témoignent la prise de position de l'Association québécoise des professeurs de français et de Radio-Canada, sur laquelle nous reviendrons. En ce qui a trait à l'association de la norme à un groupe social particulier, Barbaud (1998: 110) précise que

la condition de légitimité se réalise dans cette masse parlante lorsqu'un fait de langue est cautionné par les élites sociales, celles-ci ne manifestant aucun réflexe de rejet ou d'ostracisme à son endroit. Or, gardons-nous de restreindre les élites bourgeoises aux seules élites lettrées des milieux artistiques [...]. De nos jours, le prestige de l'argent, du savoir, de la science, de la technologie, de l'industrie du spectacle et de l'activité médiatique confère beaucoup de légitimité à un nombre considérable d'individus regroupés en diverses élites.

Toutefois, force est de constater que les descriptions de cette variété ciblée demeurent rares et les attitudes envers certaines composantes de la langue soulèvent encore aujourd'hui un bon nombre de questions.

\subsection{Norme et institutions sociales: à quoi sert la norme?}

Dès lors que nous avons précisé le type de norme qui fait l'objet de notre réflexion, il importe de se poser la question suivante: à quoi servent les normes linguistiques? Afin d'explorer des pistes de réponse, nous limitons ici notre discussion à deux sphères sociales où les questions de norme(s) semblent particulièrement pertinentes: l'éducation et les médias.

\subsubsection{La norme en éducation}

La notion de norme occupe une grande place en éducation. En effet, le personnel enseignant joue un rôle important dans le développement de la compétence (socio)linguistique des enfants. Si l'école a pour mission d'aider les petits francophones à acquérir le français "standard", une norme spécifique sera nécessairement la cible de cet enseignement. Au Québec, dès 1977, les intervenants en milieu scolaire, représentés par l'Association québécoise des professeurs de français (AQPF), s'entendaient pour dire que la norme enseignée dans les écoles devait être «le français standard d'ici». Cette variété était alors décrite comme "la variété de français socialement valorisée que la majorité des Québécois francophones tendent à utiliser dans les situations de communication formelle» (AQPF 1977). Il est donc nécessaire de documenter cette variété de façon empirique.

Dans le reste du Canada, où le français est minoritaire (p. ex., l'Ontario, l'Ouest canadien), la question des variétés à aborder dans les écoles francophones et auprès des apprenants du français langue seconde (FLS) est également d'actualité. Dans les cours d'anglais langue seconde (ALS), le modèle langagier est sans conteste nord-américain plutôt que britannique: les apprenants sont exposés aux variétés régionales et encouragés à adopter des usages (lexicaux, phonologiques, etc.) qui correspondent à la réalité sociolinguistique de l'Amérique. Or, pour le FLS, le modèle linguistique reste souvent axé sur un standard idéalisé, uniforme, une norme fantasmée que plusieurs croient, à tort, d'usage courant en France (French et Beaulieu 2016). Ce manque d'exposition aux variétés nationales amène certains apprenants à avoir une compétence sociolinguistique lacunaire dans leurs interactions orales, qui se traduit notamment par une tendance à surutiliser les formes soutenues dans des situations familières (Dewaele 2004, Mougeon et al. 2010, Thomas 2015). Pour tenter de remédier à cette absence de 
modèle national, Cox (1998) proposait une norme sociolinguistique pour l'enseignement de la phonétique française au Canada basée sur le parler des lecteurs de nouvelles de Radio-Canada, média francophone perçu à l'échelle nationale comme un modèle langagier. Bien qu'elle replace la norme dans son contexte géographique (ici le Canada), cette démarche reste fondée sur de la parole spécialisée qui ne sera pas forcément considérée comme la cible à atteindre pour les locuteurs "ordinaires", a fortiori de langue seconde, et ce, même en situation formelle.

Dans les programmes postsecondaires de formation des maîtres, la didactique de l'oral pourrait sensibiliser les étudiants inscrits à un programme de formation des enseignants aux formes linguistiques propres au mode oral, telles qu'elles sont attestées au Canada et à travers la francophonie. Par exemple, les futurs enseignants pourraient développer une meilleure connaissance du français oral par le biais d'exercices d'observation (Auger 2009); des ressources pédagogiques intéressantes sont proposées dans Detey et al. (2010), qui consacre trois chapitres à des variétés parlées dans le domaine laurentien (Québec, Ontario, Alberta). Cet apprentissage pourrait mener à une discussion sur les normes pédagogiques (Valdman 1967), qui proposent une démarche en FLS où l'on tente de tenir compte des comportements observables et des évaluations des locuteurs dans la communauté-cible, mais aussi du processus d'acquisition d'une langue seconde. L'idée ici n'est pas d'imposer un modèle unique, mais d'inclure la variation dans la progression pédagogique. Que l'apprenant décide ou non de se conformer aux usages sociolinguistiques de sa communauté, l'éveil de la conscience sociolinguistique peut amener chacun d'entre eux à mieux reconnaître et apprécier la réelle diversité du français.

\subsubsection{La norme dans les médias}

Le monde médiatique entretient également des liens particuliers avec la norme. De par son mode de transmission, la presse écrite n'a recours qu'à la morphosyntaxe du français écrit, le domaine phonique ne s'y prêtant évidemment pas. Pour observer les usages et les évaluations de la norme orale, il faut ainsi se tourner vers les médias radiophoniques et télévisuels. Sur sa norme institutionnelle, la position de Radio-Canada est explicite «le français utilisé sur les ondes de la Radio et de la Télévision françaises ainsi que dans le site Internet de Radio-Canada est le français correct en usage au Canada» (RadioCanada 2004). Le diffuseur national compte également dans son personnel un conseiller linguistique qui, en plus d'orienter la norme au sein de l'organisme, partage avec les auditeurs d'un bout à l'autre du pays des «capsules linguistiques» où il fait des propositions normatives (Remysen 2010), comme la suivante concernant la distinction entre oui et si.

L'adverbe oui sert à répondre à une question positive et l'adverbe si sert à répondre à une question négative. [...] Cette utilisation du si n'est pas très répandue chez nous. Pourtant, il s'agit d'une règle de grammaire élémentaire. En effet, toutes les grammaires françaises considèrent comme une faute l'utilisation de l'adverbe oui pour réfuter une question négative. (Bertrand 1999: 125-126)

Des orientations normatives peuvent aussi transparaître dans les décisions prises lors du doublage en français d'émissions et de films originalement en langue anglaise ou étrangère. Les travaux de Reinke et Ostiguy montrent, d'une part, que le modèle privilégié par les doubleurs est celui que l'Union des artistes décrit comme le français «international» (Reinke et Ostiguy 2012) mais, d'autre part, que les attitudes du public et des professionnels du doublage reflètent leur ambigüité face à la légitimité des usages réels tant du Québec que de la France (Ostiguy et Reinke 2015). Il n’en demeure pas moins que, en plus de donner libre cours à certaines évaluations explicites des usages, la langue des médias donne 
également accès à des données empiriques permettant d'observer les normes objectives. Des travaux en sociolinguistique ont entre autres proposé que la langue employée à la télévision reflète la diversité présente dans la société québécoise (Reinke 2005) et que l'usage soutenu des élites culturelles locales correspond en grande partie à la norme présentée dans Le Bon Usage (Bigot 2008, 2011).

\section{Norme orale et norme écrite}

Les normes prescriptives sont perçues de façon essentiellement monolithique et immuable: telle forme correspond ou ne correspond pas à la norme définie comme modèle linguistique, sans réelle possibilité d'adaptation à l'objet ou au contexte social ou historique. Cette vision unitaire pose au moins trois problèmes, reliés à la variation diaphasique (ou stylistique), à la variation diatopique (ou dialectale) et au traitement différencié des différentes composantes de la langue. La norme linguistique entretenant par ailleurs des liens particuliers avec le social, aborder «la norme orale» stimule une réflexion sur la variation diastratique (ou sociale) : la normalisation linguistique perpétue-t-elle l'hégémonie et les discriminations diverses des utilisateurs de formes exclues de «la» norme?

D'une part, un même modèle linguistique peut-il s'appliquer à tous les contextes d'usage de la langue? L'oral spontané doit-il se conformer à la même hiérarchisation des usages que l'écrit formel? D'autre part, est-il raisonnable d'établir une norme unique pour toutes les zones de la francophonie, face à des usages parfois très divergents? Cette dernière question rejoint évidemment toute la réflexion sur le développement de modèles pluricentriques du français et les tentatives de légitimation des variétés dites "régionales» (Klinkenberg 2002). Une des difficultés, largement minimisée, que soulèvent ces deux séries de questions est que les réponses varient grandement selon les composantes de la langue, qu'on peut ramener à trois: la morphosyntaxe, le lexique et la prononciation.

La vision unitariste de la norme prescriptive s'impose le plus clairement pour la morphosyntaxe. La norme morphosyntaxique s'appuie sur l'écrit formel: elle est identique à travers la francophonie et «bien» parler ne signifie pas autre chose que parler comme on écrit:

S’il est relativement facile de décider qu'il convient d'éviter une construction comme Donne-moi-le pas dans une entrevue télévisée ou qu'il est préférable de formuler ses questions à l'aide de l'inversion plutôt que du marqueur interrogatif - tu (ex., Parle-t-elle français? plutôt que Elle parle-tu français?), les choix ne sont pas toujours aussi clairs sur les plans lexical et phonologique. Doit-on aller magasiner ou faire son shopping, prendre le traversier ou le ferry? Convient-il d'éviter toute affrication dans la prononciation de mots comme tuque et dire et de faire disparaitre la distinction de longueur vocalique qui différencie des mots comme maître et mettre? (Auger 2005: 68)

La norme lexicale présente un plus grand degré de flexibilité, dans les dimensions à la fois diatopique et diaphasique. Les dictionnaires généraux incluent d'emblée des marques qui restreignent l'usage d'un mot à des régions ou à des styles de parole particuliers (p. ex., au Canada, en Savoie, familier, technique). Il n'en demeure par moins que ces marques sont porteuses d'une forme de hiérarchisation des usages. Un mot étiqueté comme "canadien", même s’il est légitimé par son inclusion à la nomenclature du dictionnaire, sera ainsi largement perçu comme subordonné à un synonyme d'usage général. De même, un mot «familier», "populaire», «argotique» ou "vulgaire» (sans qu'on perçoive clairement ce qui justifie le choix de l'un ou l'autre de ces qualificatifs) sera considéré négativement par rapport à un mot dépourvu de marque d'usage. 
Enfin, la prononciation se trouve dans une situation particulière par rapport à la morphosyntaxe et au lexique. Elle est exclusive à l'oral, ce qui la met en porte-à-faux par rapport à une norme fondée sur l'écrit. Certains dictionnaires (p. ex., le Petit Robert, mais pas le Larousse) incluent bien des transcriptions "phonétiques", mais ce qualificatif est largement trompeur puisque ces transcriptions sont essentiellement de nature phonémique. Elles se limitent donc à l'expression des oppositions - le Petit Robert oppose patte [pat] et pâte [pat], mais pas mettre et maître, tous deux transcrits [metı] - mais passent sous silence à la fois les traits de prononciation qui relèvent de la réalisation (phonétique) de ces oppositions et de phénomènes propres à la juxtaposition de mots. Ainsi, comment considérer l'ouverture des voyelles fermées [i y u] en syllabe fermée par une consonne non-allongeante en français laurentien? La liaison entre est et un mot à initiale vocalique est-elle obligatoire? Par ailleurs, les systèmes phonémiques, surtout vocaliques, varient grandement à l'intérieur de l'espace francophone, mais la norme prescriptive ne s'est pas positionnée par rapport à ces variations.

\subsection{Composante morphosyntaxique}

Malgré la vision unitariste de la norme morphosyntaxique, où «bien» parler signifie essentiellement s'exprimer comme à l'écrit formel, il demeure que les distinctions entre l'oral soutenu et l'écrit formel sont flagrantes pour certains traits linguistiques. Ainsi, si certaines formes sont facilement "admises" tant à l'oral qu'à l'écrit, et ce dans l'ensemble de la francophonie, d'autres semblent "réservées» au mode oral. On observe cette généralisation avec deux phénomènes morphosyntaxiques binaires, où l'on choisira tantôt l'une, tantôt l'autre forme selon que l'on s'exprime à l'écrit ou à l'oral.

La particule négative ne illustre la variation diamésique maximale pouvant exister entre l'oral et l'écrit: "Lorsque la négation porte sur un verbe, l'effacement de ne reste limité, surtout dans la langue écrite»(Grevisse et Goosse 2011: 1343). Les études empiriques appuient partiellement ce constat: quasi catégorique à l'écrit formel, l'emploi de ne est, depuis les années 1990, généralement inférieur à 30 \% à l'oral (Ashby 2001), y compris dans des situations de conversation plus formelle (Reinke 2005, Villeneuve 2017). Le prétendu "effacement» de ne est donc loin de "reste[r] limité» à l'oral spontané et peut difficilement continuer d'être étiqueté «familier». On observe un écart semblable pour les clitiques pronominaux désignant un sujet de première personne du pluriel, où la variante on alterne avec nous, «surtout dans la langue parlée familière» (Grevisse et Goosse 2011: 110). En effet, si on s'emploie assez rarement à l'écrit (Rehner, Mougeon \& Nadasdi 2003), son usage est généralisé à l'oral spontané (Coveney 2000, Fonseca-Greber et Waugh 2003). Il existe toutefois peu de données tirées de la parole soutenue, d'où la difficulté de tester la description du Bon Usage. Il est important de noter que dans les deux cas, bien que la forme dite «familière» ne soit pas stigmatisée même à l'oral soutenu, son emploi à l'écrit continue de faire l'objet de jugements défavorables. Si l'on observe un écart si important entre la norme fantasmée (Moreau 1997) et l'usage réel pour ces deux variables, ne et nous peuvent-ils vraiment continuer de constituer la norme prescriptive pour l'oral spontané? Si oui, quel est donc l'écart nécessaire entre les normes réelles orale et écrite avant qu'on n'accepte qu'une forme typique de l'oralité ne soit tolérée au sein de la norme de ce mode de communication?

Un dernier exemple, celui des interrogatives totales, illustre cette même dissociation entre oral et écrit, cette fois pour une variable dépendante où plus de deux variantes sont en compétition. Ainsi, selon Le Bon Usage, l'interrogation «est marquée par l'intonation dans l'oral» et par l'inversion «dans la langue écrite ou dans la langue soignée», alors que "est-ce que, tout à fait courant dans l'oral, n'est pas inconnu de la langue écrite, même soignée» (Grevisse et Goosse 2011 : 506). Les études empiriques 
soutiennent ces généralisations, montrant clairement le déclin de l'inversion, particulièrement en France (Coveney 1996/2002, Elsig 2009, Farmer 2015). Les variétés hexagonale et québécoise semblent toutefois se distinguer sur le plan diaphasique en ce qui a trait à la valeur stylistique de certaines variantes, comme on le verra plus loin.

Bref, bien que la vision unitariste de la norme distingue relativement peu la morphosyntaxe de l'écrit et de l'oral soutenu, il semble que toute discussion de la norme orale doive tenir compte de la variation diamésique.

\subsection{Composante phonique}

La nature exclusivement orale des aspects phoniques de la langue établit le caractère nécessairement incomplet d'une norme basée sur la langue écrite, puisque seule une norme orale parvient à documenter la prononciation. Il ne faut pas pour autant en conclure que l'écrit n'est pas pertinent pour la définition d'une norme de prononciation. On peut en effet distinguer deux types de variables phoniques, selon qu'elles se reflètent ou non dans la forme orthographique. La qualité des segments n'est le plus souvent pas reflétée à l'écrit. Par exemple, la forme écrite n'indique pas si la voyelle de mètre est distincte de celle de mettre (elle l'est au Canada mais pas dans d'autres variétés), si la voyelle de saute peut ou non être diphtonguée, si le $<\mathrm{t}>$ de tuque peut être assibilé ou quels lieu et mode d'articulation le $<\mathrm{r}>$ doit prendre dans différents contextes segmentaux et prosodiques.

Par contre, la forme écrite des mots étant perçue comme la forme "complète», toute prononciation qui s'en écarte par l'omission ou l'ajout d'un segment ou une modification de l'ordre des segments tend à être considérée d'emblée comme fautive. La correspondance avec la forme orthographique mène ainsi au rejet de la métathèse dans [areopor] pour aéroport ou de l'épenthèse dans exprès [Eksipre], tout en favorisant l'acceptation de prononciations comme sculpter [skylpte], avec un [p] absent de la forme historique mais justifié par l'orthographe, illustration de l'effet Buben (Buben 1935, Chevrot et Malderez 1999). Dans la plupart des cas d'écart entre l'oral et l'écrit, il s'agit cependant de l'omission à l'oral d'un segment ayant un correspondant à l'écrit. Ainsi, si certains groupes de consonnes en fin de mot sont régulièrement simplifiés à l'oral par l'omission de la dernière consonne (Côté 2004) - c'est le cas notamment des groupes obstruante-liquide (p. ex., livre prononcé [liv]) - de telles formes, courantes dans l'ensemble des variétés de français, seront vues comme extérieures à la norme prescriptive. On peut également mentionner dans cette catégorie de variables phoniques l'omission du [1] des déterminants définis et des clitiques objets la et les (p. ex. à la place [a:plas]), plus courante en français laurentien mais pas exclusive à cette variété (Ashby 1984, Poplack et Walker 1986).

La liaison et le schwa constituent des cas particuliers de réalisation ou d'omission segmentale. De façon générale, les formes avec liaison et schwa seront perçues comme plus soutenues que les alternatives sans liaison ou schwa (p. ex., est $[\mathrm{t}]$ allé > est $\varnothing$ allé; cerise [sœriz] > [sriz]), mais seulement si ces segments correspondent à une lettre dans la forme écrite. Ainsi, si des formes comme les jours [z] arrivent, avec liaison entre un sujet nominal et un verbe, ne seront pas exclues de la norme, même si elles sont complètement absentes de l'oral (Durand et al. 2011), des séquences courantes comme vingt [z] amis seront condamnées étant donnée l'absence d'une consonne écrite correspondant au [z] de liaison. C'est à ce titre que le [1] intervenant entre ça et un verbe à initiale vocalique en français laurentien (p. ex., ça [1] arrive) est lourdement stigmatisé, alors que ce [1] peut aisément être analysé comme une consonne de liaison analogue au [n] de on [n] arrive (Lyche et al. 2018). Mais même si on se restreint aux consonnes de liaison présentes à l'écrit, il est clair que la question de la norme est loin d'être résolue, entre des 
contextes où la consonne est catégorique et d'autres où elle est pratiquement exclue dans l'usage. La situation du schwa est quelque peu différente puisqu'une dissociation tend à s'opérer entre la voyelle écrite <e> et la voyelle orale [œ] ou [ø] (le timbre dépendant des variétés et des contextes). À l'intérieur des mots, seules les voyelles orthographiques sont permises dans la prononciation (ex. tenu [t(œ)ny] vs pneu [pnø] mais pas [pœnø]). En fin de mot, par contre, tout groupe consonantique final peut être suivi d'un schwa à l'oral, sans égard à la présence d'un <e> orthographique (p. ex., un film, on filme).

L'ensemble de ces éléments montre bien qu'il y a lieu de clarifier les liens entre la norme de prononciation et l'orthographe, tant dans l'usage que dans les principes qu'on pourrait vouloir établir.

\section{Une norme orale en français laurentien?}

Dans le domaine laurentien, les débats sur la norme, en partie symbolisés par l'opposition entre les endogénistes, promoteurs d'une norme québécoise, et les exogénistes (Bouchard 1998), défenseurs d'une norme hexagonale ou «internationale», ont essentiellement porté sur le lexique. La prononciation a été abordée de façon marginale, alors que la vision unitariste de la morphosyntaxe, elle, n’est pas remise en question.

\subsection{Composante morphosyntaxique}

La variation diatopique est relativement peu documentée pour la morphosyntaxe, mises à part quelques descriptions sommaires. Si l'on a pu déterminer que des constructions impératives telles que dis-moile (plutôt que dis-le-moi) ou l'emploi de la particule -tu dans les interrogatives totales (p. ex., Il est-tu arrivé? plutôt que Est-il arrivé?) seraient exclues de la norme orale (Auger 2005), il est plus difficile d'évaluer la place d'autres constructions morphosyntaxiques. En effet, on peut imaginer des cas où des formes typiquement laurentiennes seraient attestées dans l'oral soutenu, là où les locuteurs seraient relativement peu conscients de la variation diatopique avant d'être confrontés à des normes exogènes. Par exemple, il est difficile de déterminer si les infinitives hypothétiques (p. ex., Avoir su, je serais partie plutôt que Sijavais su, je serais partie), attestées en français québécois mais pas en français hexagonal (Martineau et Motapanyane 1996), pourraient être incluses dans une description de la norme. Une meilleure documentation de l'oral laurentien soutenu serait nécessaire pour mesurer l'usage et l'évaluation de telles formes.

Des formes attestées à l'oral de part et d'autre de l'Atlantique peuvent aussi être sujettes à des différences dialectales de nature quantitative (fréquence d'usage) ou diaphasique (évaluation "familière" ou «soutenue»). D’une part, la référence temporelle au futur illustre bien qu'une variante associée à la formalité à travers la francophonie puisse être employée à des fréquences différentes dans diverses régions. En effet, hormis les contextes négatifs, la variante synthétique (p. ex., tu chanteras) est peu employée dans le domaine laurentien, même en situation de communication formelle ou chez des locuteurs plus éduqués (Bigot 2011, Wagner et Sankoff 2011, Villeneuve et Rosychuk 2017), mais elle se maintient assez bien en France, surtout en cooccurrence avec d'autres variantes soutenues (Roberts 2012). D'autre part, la variation dans les interrogatives totales permet de montrer que la variation diatopique peut porter non pas sur la fréquence, mais sur l'association d'une même variante à des styles différents. Ainsi, si l'inversion semble généralement associée à l'écrit et à la formalité ${ }^{6}$, la variante est-ce

6. En français québécois, les interrogatives comportant un sujet de $2^{\mathrm{e}}$ personne, un contexte où l'inversion est favorisée peu importe le niveau de formalité, constituent une exception à cette généralisation. 
que, surtout associée au français familier en France, marque plutôt la formalité dans le domaine laurentien (Elsig et Poplack 2006).

Ainsi, à moins de disposer de descriptions détaillées de l'usage soutenu dans le domaine laurentien, il est difficile d'imaginer comment les ouvrages pédagogiques, entre autres les manuels de FLS, pourront aborder de façon nuancée la norme morphosyntaxique qui a cours à l'oral.

\subsection{Composante phonique}

Sur le plan phonique, contrairement à la morphosyntaxe, la variation diatopique est bien établie et bien documentée. La question de l'intégration de cette variation dans la norme orale reste cependant largement en suspens. L’Office de la langue française (1965: 6) écrivait: «Quant à la phonétique [...], la marge de variation doit être minime et ne tenir compte que de très légères différences d'accent [...].» Quarante ans plus tard, Radio-Canada (2004) précisait: "Les prononciations utilisées à la Radio et la Télévision française de Radio-Canada doivent se rapprocher le plus possible des prononciations en usage dans le reste de la francophonie.» De façon générale, on n’entend donc pas favoriser un modèle de prononciation laurentien, mais il faut admettre que les "très légères différences d'accent» et les "prononciations en usage dans le reste de la francophonie» ne sont nulle part précisées. Les usages dans la francophonie étant eux-mêmes variables, l'étalon qui pourrait servir à mesurer les différences d'accent ou à servir de cible reste flou.

Plus récemment, USITO (Cajolet-Laganière et Martel 2013) adoptait comme principe de transcription phonétique qu'elle soit

fondée sur les faits réels de la prononciation du français québécois standard telle qu’on peut l'observer dans les manifestations officielles de la parole publique, essentiellement dans les émissions d'information et d'affaires publiques des réseaux publics (non commerciaux) de radio et de télévision.

L'approche n'est donc plus directement différentielle, par rapport à d'autres variétés de français, mais basée sur l'usage local. En cela, la démarche d'USITO rejoint les études descriptives de Cox (1998) et de Reinke (2005), même si elle ne s'appuie pas sur un corpus défini.

Plusieurs variantes de prononciation spécifiquement laurentiennes ont ainsi fait l'objet de positionnements par rapport à la norme. Les oppositions vocaliques historiques, en régression ailleurs dans la francophonie mais encore stables au Canada, offrent le cas le plus clair d'intégration dans une norme laurentienne. La question a surtout porté sur l'opposition entre les deux voyelles nasales de brin et brun et les deux voyelles mi-ouvertes de faites et fête, étant acquis que les deux voyelles ouvertes de patte et pâte et les deux séries de voyelles moyennes [e ø o] et [ $\varepsilon$ œ o] (épée vs épais, jeûne vs jeune, côte vs cote) restent bien distinctes dans toutes les variétés laurentiennes et tous les styles de parole. À l'exception de l'opposition faites-fête, les ouvrages de référence des deux côtés de l'Atlantique conservent de toute façon ces oppositions dans leurs transcriptions et l'étude de Cox (1998) confirme le maintien des contrastes brin-brun et faites-fête chez les lecteurs de nouvelles de Radio-Canada. Cette dernière opposition est par ailleurs entérinée dans les transcriptions du dictionnaire USITO, qui reconnaît également des différences de durée pour les voyelles fermées, pool [pu:l] et jeans [dzi:n] s'opposant par exemple à poule [pul] et gin [dzin].

Les variantes laurentiennes considérées comme allophoniques génèrent un continuum évaluatif allant de l'exclusion claire de la norme à une acceptation plus ou moins appuyée. La majorité des traits tombent dans la première catégorie, qui inclut la diphtongaison des voyelles longues, les variantes 
en [we] ou [we] de la diphtongue <oi >, le $\mathrm{R}$ apical et d'autres traits considérés comme marqués (Cox 1998, Reinke 2005, Chalier 2018). À l'autre extrémité, l'assibilation ou affrication de [t d] devant voyelles antérieure fermée est sans doute le trait le mieux intégré à la norme (Cox 1998), considéré comme «socialement neutre» par USITO (Cajolet-Laganière et Martel 2013). Dans une position intermédiaire, on note l'ouverture ou le relâchement des voyelles fermées en syllabe finale fermée par une consonne autre que [ $\mathrm{v} \mathrm{z}$ 3], le timbre antériorisé des voyelles nasales [ã] et [ẽ] et le timbre de la voyelle ouverte [a] ou [a] en fin de mot. Des deux premiers phénomènes, Reinke (2005: 20) dit qu'ils ne sont pas "perçu[s] par les Québécois comme étant des prononciations témoignant d'une mauvaise qualité de langue», alors que l'ouverture des voyelles fermées n'est "pas socialement neutre» selon USITO (voir aussi Dumas 1987). Quant au timbre des voyelles ouvertes en fin de mot, si Reinke (2005) et USITO admettent la postérieure [a], Cox penche pour [a], tout comme le Multidictionnaire (De Villers 2015). On néglige pourtant dans le débat sur les voyelles fermées et le choix entre [a] et [a] le fait que le relâchement (pour les voyelles fermées) et le lieu d'articulation (pour les voyelles ouvertes en fin de mot) peuvent aussi avoir une valeur contrastive: une bise [biz] ne peut être confondu avec le chanteur Biz [brz] (du groupe Loco Locass) ou avec un quiz [kwIz], ni $\grave{a}, m a, l a$ [a, ma, la] avec $a$, $m^{\prime} a$, là [a, ma, la]. De ce fait, le relâchement et le lieu d'articulation acquièrent un statut qui n'est pas simplement stylistique et qui devrait éclairer la discussion.

La variation diatopique dans le domaine de la prononciation ne se limite pourtant pas à des variantes spécifiques qu'il convient d'évaluer. Comme pour la référence temporelle au futur et les interrogatives totales en morphosyntaxe, certaines variantes phoniques communes aux variétés européennes et américaines peuvent aussi différer dans leur fréquence d'usage et dans la façon dont elles sont perçues par les locuteurs. Comparons par exemple la réduction des groupes consonantiques et l'insertion de schwa en fin de mot, deux stratégies concurrentes pour résoudre les groupes consonantiques complexes, dans différentes variétés de français. Milne (2014) montre que la réduction des groupes est significativement plus fréquente, dans des styles de parole avec différents degrés de formalité, chez les locuteurs québécois que chez les locuteurs français, alors que l'insertion de schwa suit la tendance inverse. En conséquence, on peut raisonnablement penser que l'évaluation sociolinguistique de ces deux processus - et donc leur relation à la norme de prononciation - sera différente dans les deux communautés. Ces questions restent cependant largement inexplorées.

\subsection{Contributions au numéro}

Les contributions au présent numéro, qui proviennent de chercheurs chevronnés et émergents, posent la question de la norme en français oral en contexte laurentien, bien que la plupart soient basées sur des données spécifiquement québécoises. Elles abordent la question de la norme à la fois sous l'angle prescriptif, objectif et évaluatif.

Dans le premier article (Regard rétrospectif sur la norme du français québécois oral), Bigot propose une synthèse de certains débats sur la norme orale en français québécois. Il revient d'abord sur les différents modèles - «international», «québécois» et «radio-canadien» - qui s'opposent dans l'établissement d'une norme et sur la définition des locuteurs sélectionnés comme représentatifs de cette norme. Il fait ensuite le point sur les éléments descriptifs qui caractérisent potentiellement la norme québécoise orale, appréhendée à partir de corpus de parole médiatique. Il conclut que la grammaire de l'oral normé se distingue peu de celle de l'écrit, telle que consignée dans les ouvrages de référence comme le Bon Usage de Grevisse-Goose. À l'opposé, la description de la norme de prononciation reste 
incomplète, en raison du statut incertain de plusieurs traits typiques des variétés laurentiennes et du fait que la prononciation n'a jusqu’à présent été étudiée que dans la parole spécialisée de lecteurs de nouvelles, et non pas généralement de celle des élites québécoises en situation de communication formelle. Bigot conclut à la nécessité d'un guide descriptif complet sur la norme du français québécois oral, à des fins notamment pédagogiques.

Côté et Remysen (Le "bon usage du français au Québec " selon le Multidictionnaire de la langue française: le cas de la prononciation) s'intéressent aux notes de prononciation figurant dans la nomenclature du Multidictionnaire de Marie-Éva de Villers, ouvrage qui s'est rapidement imposé comme référence en matière de norme prescriptive au Québec. Ces notes concernent les mots jugés comme présentant des difficultés de prononciation, qui représentent environ $5 \%$ de la nomenclature. Côté et Remysen passent en revue à la fois les stratégies utilisées pour rendre la prononciation d'un mot et les traits de prononciation ciblés par ces notes. Ces notes sont alors analysées par rapport à l'objectif avoué du Multidictionnaire de décrire le bon usage du français au Québec. La comparaison entre les recommandations du Multi, les transcriptions du Petit Robert et les prononciations courantes au Québec montre cependant que le Multi accorde très peu de légitimité à l'usage québécois, ce qui confirme pour la prononciation les orientations déjà notées pour le lexique.

Le texte de Villeneuve (Normes objectives et variation socio-stylistique: le français québécois parlé en contexte d'entrevues télévisées) aborde plutôt le problème de la norme objective telle qu'elle se présente dans la parole de membres des élites culturelles et politiques québécoises en situation d'entrevue. Après la présentation d'un corpus télévisuel permettant d'examiner la variation diastratique et diaphasique en parole soutenue, Villeneuve teste plus avant dans quelle mesure l'oral normé représente réellement l'écrit. Par le biais d'une analyse variationniste de l'alternance des auxiliaires et de la négation verbale, elle montre que la conformité de l'oral soutenu à la norme écrite n'est pas constante pour toutes les variables morphosyntaxiques binaires et dépend notamment du niveau de stigmatisation sociale de la forme dite «familière». Si l'absence de la particule de négation ne passe aujourd'hui inaperçue à l'oral, même dans la conversation formelle, l'emploi de l'auxiliaire avoir demeure marginal en situation d'entrevue télévisée. La documentation de la variation diaphasique dans la parole spontanée fait donc ressortir les nuances nécessaires à une meilleure compréhension du continuum familier-soutenu à l'oral spontané.

Les deux textes suivants abordent la norme sous un angle évaluatif, décrivant les attitudes d'apprenants du FLS en contextes québécois envers des variantes québécoises. Par le biais d'une tâche d'écoute employant la technique du locuteur masqué ("matched guise technique»), Guertin (Variation sociophonétique dialectale et stylistique: quelle est la langue cible en français langue seconde à Montréal?) évalue la perception qu'ont des apprenants adultes vivant à Montréal d'extraits de parole provenant de locutrices françaises et québécoises dans des styles formel (ou soutenu) et informel (ou familier). Deux questions ont été posées, sur l'origine géographique perçue des extraits - France ou Québec - et sur l'adéquation entre les extraits et la langue cible valorisée par les apprenants. D'une part, on constate un amalgame partiel entre le style et l'origine géographique: les apprenants ont tendance à associer les extraits formels à la variété française et les extraits informels à la variété québécoise. D’autre part, la langue cible préférée des apprenants correspond aux extraits formels davantage qu'à une variété géographique particulière. Conjointement, ces résultats suggèrent que le français québécois reste perçu moins positivement et que les représentations linguistiques n'accommodent pas encore pleinement la notion de norme québécoise formelle.

Enfin, le texte de Beaulieu, French et Gagnon ("I'veulent-tu parler comme nous-autres?» Opinions d'apprenants sur la forme interrogative totale en français laurentien) ajoute des considérations 
pédagogiques à la dimension évaluative en mesurant l'effet d'un enseignement explicite de la variation sur la perception des apprenants d'une structure interrogative typique en français québécois: la particule interrogative postverbale $-t u$. Après la présentation et la mise en pratique (en perception) de différentes formes de l'interrogation totale en français dans trois classes de FLS de niveau avancé, les apprenants ont eu à répondre à un court questionnaire portant notamment sur leur utilisation de l'interrogation en $-t u$ et l'usage qu'ils projetaient faire des différentes formes interrogatives. Les résultats montrent que la sensibilisation à l'interrogation en $-t u$ a eu un impact positif sur l'usage et la perception de cette variante régionale: plus de la moitié des participants prévoient faire usage de l'interrogation en $-t u$ et même ceux qui ne le souhaitent pas le font pour des raisons stratégiques et utilitaires et non parce qu'ils considèrent cette forme comme fautive. Cette étude montre la pertinence de la notion de réflexion métasociolinguistique, qui pousse plus avant le concept de réflexion métalinguistique de Gombert (1992) en se focalisant sur «la diversité des usages de la langue et leurs impacts sur la communication».

\section{Conclusion}

À l'issu de ce numéro thématique, de nombreuses questions subsistent. D'abord, au plan diachronique, on constate clairement le côté mouvant de toute discussion portant sur la norme orale: ses deux composantes - l'objectif et le subjectif - sont en constante évolution. Ainsi, plus de 40 ans après la Révolution Tranquille, est-il toujours pertinent de parler de «la variété de français socialement valorisée que la majorité des Québécois francophones tendent à utiliser dans les situations de communication formelle», comme le faisait l'AQPF dans les années 70? Devant une francophonie québécoise de plus en plus diversifiée, quels "Québécois francophones» serviraient aujourd'hui de modèles linguistiques? Ensuite, au plan diatopique, un même modèle linguistique, basé par exemple sur l'usage soutenu du français parlé au Québec, peut-il s'appliquer à toutes les zones du domaine laurentien? Le fait que la totalité des contributions reçues pour une numéro sur "la norme orale en français laurentien " portaient sur la variété québécoise témoigne-t-il déjà de sa domination sociolinguistique vis-à-vis des francophonies minoritaires de l'Ontario et de l'Ouest canadien? Dans le cadre d'une réflexion sur la norme orale, quelle place faudrait-il désormais accorder aux normes locales? Enfin, et surtout, au plan diastratique, quel rôle la norme dominante joue-t-elle dans le renforcement de l'insécurité linguistique? Si la norme continue de maintenir l'hégémonie sociolinguistique et de perpétuer les inégalités sociales en sanctionnant l'usage des groupes sociaux dominants (Aléong 1983), faudrait-il plutôt remettre en question l'existence même de normes? Sans prétendre proposer de réponse à ces préoccupations de nature sociologique, on peut tout de même se demander s'il serait plus fructueux de consacrer davantage d'attention à une sensibilisation à la variation (diastratique, diaphasique et diatopique) afin de favoriser le développement d'une francophonie locale, régionale et globale plus vivante et assurée.

\section{Références bibliographiques}

Aléong, S. 1983. Normes linguistiques, normes sociales, une perspective anthropologique La norme linguistique (p. 255-280). Québec: Conseil supérieur de la langue française.

Ashby, W. 1984. "The elision of /1/ in French clitic pronouns and articles». Michigan Romance studies 1 (11): $1-16$. 
Ashby, W. J. 2001. «Un nouveau regard sur la chute du ne en français parlé tourangeau: S'agit-il d'un changement en cours». Journal of French Language Studies 11 (1): 1-22. doi: http://dx.doi.org/10.1017/ S0959269501000114.

Association québécoise des professeurs de français [AQPF]. (1977). "Le discours de M. Jacques-Yvan Morin: L’enseignement du français ». Québec français (28) : 13-15.

Auger, J. (2005). "Un bastion francophone en Amérique du Nord: le Québec». Dans A. Valdman, J. Auger et D. Piston-Hatlen (dir.), Le français en Amérique du Nord: État présent (p. 39-79). Québec: Presses de l'Université Laval.

Auger, J. 2009. Langue parlée et enseignement: Quelques idées en vue d'un cours de linguistique destiné aux futurs enseignants. Dans R. Bergeron, G. Plessis-Bélair et L. Lafontaine (dir.), La place des savoirs oraux dans le contexte scolaire d'aujourd'hui (p. 11-32). Québec: Presses de l'Université du Québec.

Barbaud, P. 1998. «Dissidence du français québécois et évolution dialectale». Revue québécoise de linguistique 26 (2) : 107-128. doi : http://dx.doi.org/10.7202/603156ar.

Bédard, É. et Maurais, J. (dir.). 1983. La norme linguistique. Québec: Gouvernement du Québec; Conseil de la langue française.

Bell, A. 1984. «Language style as audience design». Language in Society 13 (2): 145-204. doi: http://dx.doi. org/10.1017/S004740450001037X.

Bertrand, G. 1999. 400 capsules linguistiques, ( $1^{\mathrm{er}}$ tome). Montréal: Lanctôt Éditions.

Bigot, D. 2008. "Le Point» sur la norme grammaticale du français québécois oral. (Ph.D.), Université du Québec à Montréal, Montréal.

Bigot, D. 2011. «De la norme grammaticale du français parlé au Québec». Arborescences 1. doi : http://dx.doi. org/10.7202/1001939ar.

Bigot, D. et R. A. Papen. 2013. «Sur la "norme” du français oral au Québec (et au Canada en général) ». Langage et société 146: 115-132. doi : http://dx.doi.org/10.3917/ls.146.0115.

Bloomfield, L. 1927. «Literate and illiterate speech». American Speech 2 (10) : 432-439.

Bouchard, C. 1998. La langue et le nombril: histoire d'une obsession québécoise. Montréal: Fides.

Buben, V. 1935. Influence de l'orthographe sur la prononciation du français moderne. Genève: Droz.

Cajolet-Laganière, H. et Martel, P. 1995. La qualité de la langue au Québec (Vol. 18). Québec: Institut québécois de recherche sur la culture.

Cajolet-Laganière, H. et Martel, P. 2013. USITO: parce que le français ne s’arrête jamais. Sherbrooke: Éditions Delisme. URL: https://www.usito.com.

Chalier, M. (2018). "Quelle norme de prononciation au Québec? Attitudes, représentations et perceptions». Langage et société 163 : 121-144.

Chevrot, J.-P. et Malderez, I. 1999. «L'effet Buben: de la linguistique diachronique à l'approche cognitive (et retour) ». Langue française: $104-125$.

Cooper, R. P. et Aslin, R. N. 1990. «Preference for infant-directed speech in the first month after birth». Child development 61 (5): 1584-1595.

Côté, M.-H. 2004. "Consonant cluster simplification in Québec French». Probus 16: 151-200.

Coveney, A. 1996/2002. Variability in spoken French : A sociolinguistic study of interrogation and negation. Bristol: Elm Bank Publications.

Coveney, A. 2000. "Vestiges of nous and the 1st person plural verb in informal spoken French». Language Sciences $22(4): 447-481$.

Cox, T. B. 1998. "Vers une norme pour un cours de phonétique française au Canada». Canadian Modern Language Review / Revue canadienne des langues vivantes 54 (2) : 172-197. doi : http://dx.doi.org/10.3138/ cmlr.54.2.172.

De Villers, M.-É. 2003. Multidictionnaire de la langue française (6e édition). Montréal: Québec Amérique.

Detey, S., Durand, J., Laks, B. et Lyche, C. 2010. Les variétés du français parlé dans l'espace francophone: Ressources pour l'enseignement. Paris: Ophrys. 
Dewaele, J.-M. 2004. "The acquisition of sociolinguistic competence in French as a foreign language: An overview». Journal of French Language Studies 14 (3): 301-319. doi: http://dx.doi.org/10.1017/ S0959269504001814.

Dubois, J., Giacomo, M., Guespin, L., Marcellesi, C., Marcellesi, J.-B. et Mével, J.-P. 2007. Linguistique \& sciences du langage: Grand dictionnaire. Paris: Larousse.

Dumas, D. 1987. Nos façons de parler: Les prononciations en français québécois. Sillery, QC: Presses de l'Université du Québec.

Durand, J., Laks, B., Calderone, B. et Tchobanov, A. 2011. "Que savons-nous de la liaison aujourd'hui?». Langue française (1): 103-135.

Elsig, M. 2009. Grammatical variation across space and time: The French interrogative system (Vol. 3). Amsterdam: John Benjamins.

Elsig, M. et Poplack, S. 2006. "Transplanted dialects and language change: Question formation in Québec». Penn Working Papers in Linguistics 12 (2 (Selected Papers from NWAV 34)) : 77-90.

Farmer, K. L. 2015. Sociopragmatic variation in yes/no and wh-interrogatives in hexagonal French: A real-time study of French films from 1930 to 2009. (Ph.D.), Indiana University, Bloomington, IN.

Ferguson, C. A. 1983. "Sports announcer talk: syntactic aspects of register variation». Language in Society 12 (2) : 153-172.

Fonseca-Greber, B. et Waugh, L. R. 2003. "On the radical difference between the subject personal pronouns in written and spoken European French». Language and Computers 46: 225-240.

French, L. M. et Beaulieu, S. 2016. «Effects of sociolinguistic awareness on French L2 learners' planned and unplanned oral production of stylistic variation». Language Awareness 25 (1-2): 1-17. doi : http://dx.doi. org/10.1080/09658416.2015.1122024.

Gadet, F. 1995. «Norme, variation, évaluation». Histoire Épistémologie Langage 11 (1) : 18-22.

Gombert, J. E. 1992. Metalinguistic development. Chicago: University of Chicago Press.

Grevisse, M. et Goosse, A. 2011. Le bon usage: Grammaire française (15 édition). Paris: Duculot.

Klinkenberg, J.-M. 2002. "La légitimation de la variation linguistique». L'Information grammaticale 94 (1): 22-26. doi: http://dx.doi.org/10.3406/igram.2002.2667.

Labov, W. 1972. Sociolinguistic patterns. Philadelphia: University of Pennsylvania Press.

Lyche, C., Côté, M.-H. et Durand, J. 2018. «Liaison et corpus: retour sur quelques controverses». Dans M.-H. Côté, J. Durand, C. Lyche et J. Peuvergne (dir.), Dynamiques linguistiques: variation, évolution et cognition (p. 113-138). Nanterre: Presses Universitaires de Paris Nanterre.

Martel, P. et Cajolet-Laganière, H. 1996. Le français québécois: usages, standard et aménagement. Québec: Institut québécois de recherche sur la culture.

Martineau, F. et Motapanyane, V. 1996. Hypothetical infinitives and crosslinguistic variations in continental and Québec French. Dans J. Black et V. Motapanyane (dir.), Micro-parametric syntax and dialect variation (p. 145-168). Amsterdam: John Benjamins.

Milne, P. 2014. The variable pronunciations of word-final consonant clusters in a force aligned corpus of spoken French. Thèse de doctorat, Université d'Ottawa.

Moreau, M.-L. 1997. «Types de normes». Dans M.-L. Moreau (dir.), Sociolinguistique: Les concepts de base (p. 218-223). Liège: Mardaga.

Mougeon, R., Nadasdi, T. et Rehner, K. 2010. The sociolinguistic competence of immersion students. Bristol; Buffalo: Multilingual Matters.

Office de la langue française. 1965. Norme du français parlé et écrit au Québec. Québec: Ministère des affaires culturelles du Québec.

Office québécois de la langue française. 2004. Politique de l'officialisation linguistique. URL: http://www.oqlf. gouv.qc.ca/ressources/bibliotheque/officialisation/politique_officialisation_20080425.pdf.

Ostiguy, L. et Reinke, K. 2015. La langue du doublage québécois: un français «idéal» fabriqué au Québec Migration, Regionalization, Citizenship: comparing Canada and Europe (p. 231-252. Wiesbaden: Springer. 
Ostiguy, L. et Tousignant, C. 2008. Les prononciations du français québécois: normes et usages. Montréal : Guérin universitaire.

Poplack, S. et Walker, D. C. 1986. Going through /1/ in Canadian French. Dans D. Sankoff (dir.), Diversity and diachrony (p. 173-198). Amsterdam; Philadelphia: John Benjamins.

Radio-Canada. 2004. La qualité du français à Radio-Canada: principes directeurs. URL: http://ici.radio-canada. $\mathrm{ca} / \mathrm{radio} /$ francaismicro/politique.pdf.

Rehner, K., Mougeon, R. et Nadasdi, T. 2003. "The learning of sociolinguistic variation by advanced FSL learners: The case of nous versus on in immersion French». Studies in Second Language Acquisition 25 (1): 127-156. doi: http://dx.doi.org/10.1017/S0272263103000056.

Reinke, K. 2005. La langue à la télévision québécoise: aspects sociophonétiques. Gouvernement du Québec, Office québécois de la langue française.

Reinke, K. et Ostiguy, L. 2012. «Doublage et sociolinguistique: une étude comparative du doublage québécois et français». Zeitschrift für Kanada-Studien 32: 26-48.

Remysen, W. 2010. La politique linguistique des médias publics au Québec et en Flandre: De quelle conception de la langue est-il question? Dans W. Remysen et D. Vincent (dir.), Hétérogénéité et homogénéité dans les pratiques langagières: Mélanges offerts à Denise Deshaies (p. 115-150). Québec: Presses de l'Université Laval.

Roberts, N. S. 2012. «Future Temporal Reference in Hexagonal French». University of Pennsylvania Working Papers in Linguistics 18 (2): Article 12.

Rochet, B. 1994. Le français à l'ouest de l'Ontario. Tendances phonétiques du français parlé en Alberta. Dans C. Poirier (dir.), Langue, espace, société: Les variétés du français en Amérique du Nord. Québec: Presses de l'Université Laval.

Thomas, A. 2015. «Nous/on: De la réalité linguistique à la salle de classe». Arborescences: Revue d'études françaises (5): 126-138.

Valdman, A. 1967. "Norme pédagogique: Les structures interrogatives du français». International Review of Applied Linguistics in Language Teaching 5 (1): 3-10.

Villeneuve, A.-J. 2017. "A French spoken norm under the Radio-Canada spotlight: Verbal negation and Québec cultural elites». Penn Working Papers in Linguistics 23 (2): Article 14.

Villeneuve, A.-J. et Rosychuk, D. 2017. Une norme en deux espaces: les élites culturelles québécoises à RadioCanada. Communication au colloque annuel de l'Association for French Language Studies meeting. Glendon College, Toronto, Ontario.

Wagner, S. E. et Sankoff, G. 2011. "Age grading in the Montréal French inflected future». Language Variation and Change 23 (3): 275-313. doi :1http://dx.doi.org/0.1017/s0954394511000111. 